\title{
Hard Decision Combining-based Cooperative Spectrum Sensing in Cognitive Radio Systems
}

\author{
Seung-Hwan Lee \\ School of Electrical Engineering and \\ INMC \\ Seoul National University \\ Kwanak P. O. Box 34, Seoul 151-600 Kwanak P. O. Box 34, Seoul 151-600 Kwanak P. O. Box 34, Seoul 151-600 \\ Korea \\ leesh80@ttl.snu.ac.kr \\ Dong-Chan Oh \\ School of Electrical Engineering and \\ INMC \\ Seoul National University \\ Yong-Hwan Lee \\ School of Electrical Engineering and \\ INMC \\ Seoul National University \\ Korea \\ mac81@ttl.snu.ac.kr \\ Korea \\ ylee@snu.ac.kr
}

\begin{abstract}
In this paper, we propose a cooperative spectrum sensing scheme to maximize the detection probability in cognitive radio systems. We first analytically derive a closed-form expression for the detection and false alarm probability in terms of the number of cooperating users, and then determine the number of cooperating users that maximizes the detection probability for a given false alarm probability. It is shown that the detection probability of cooperative sensing is maximized when the cooperation is performed among users with high signal-to-noise power ratio and interference-to noise-power ratio. Finally, the analytic results are verified by computer simulation.
\end{abstract}

\section{Categories and Subject Descriptors}

B.8.2 [Performance and Reliability]: Analysis and Design Aids cooperative channel sensing, cognitive radio

\section{General Terms}

Algorithms, Performance, Experimentation

\section{Keywords}

Cognitive radio, cooperation channel sensing, feedback error

\section{INTRODUCTION}

As the demand for wireless communications increases, the spectrum scarcity has become a major issue for service providers to deploy new services or enhance the capacity of existing services. Recently, cognitive radio has been under active consideration to deal with conflict between the spectrum demand and spectrum utilization [1], [2]. It is required for cognitive radio users to accurately detect the presence of primary users' signal in frequency band before the utilization. Desired spectral holes and under-utilized frequency bands can be found by means of a spectrum sensing technique.

Permission to make digital or hard copies of all or part of this work for personal or classroom use is granted without fee provided that copies are not made or distributed for profit or commercial advantage and that copies bear this notice and the full citation on the first page. To copy otherwise, or republish, to post on servers or to redistribute to lists, requires prior specific permission and/or a fee. IWCMC'09, June 21-24, 2009, Leipzig, Germany. Copyright (C) 2009 ACM 978-1-60558-569$7 / 09 / 06 \ldots \$ 5.00$
A number of single-user spectrum sensing methods have been proposed [3], [4]. The use of a matched filter can provide optimum performance with prior knowledge on the primary user signal [3]. Cyclostationary feature detection can detect the signal in very low interference-to-noise power ratio (INR) condition, but it still requires some prior knowledge on the primary user [3]. Energy detection is simple to implement, but it may suffer from the presence of fading or shadowing [4]. Without prior knowledge on the primary user, the sensing performance of a single-user spectrum sensing method may significantly deteriorate in deep fading environments [4].

This problem can be alleviated by employing a cooperative spectrum sensing technique that can achieve so-called multi-user diversity (MUD) gain [5], [6]. The base station (BS) receives the information of individual measurements (e.g., the energy of the received signal) from all secondary (or unlicensed) users and then it makes a decision by comparing a sum of the measured energy to a pre-determined threshold (i.e., by means of soft-decision combining) [5]. However, it may not be applicable to practical systems mainly due to a large amount of feedback signaling overhead. This feedback signaling overhead can substantially be reduced by detecting the spectrum in a cooperative manner using a single-bit decision on the occupancy of the spectrum, called hard-decision combining [6]. This method can provide asymptotically optimum performance as the number of users goes to infinity provided that the INRs of all users are the same [6]. However, this assumption may not be realistic since the INR of individual users may significantly vary depending on the user location. Moreover, most of previous works assume that the decision information is perfectly reported to the BS without an error.

In this paper, we consider cooperative spectrum sensing in a harddecision combining manner, assuming that users experience different INR and signal-to-noise power ratio (SNR). We first represent the detection and false alarm probability in a closed form according to the number of cooperating users. For ease of mathematical tractability, we consider the use of an OR fusion rule with energy detection. We maximize the detection probability by finding the optimum number of cooperating users for a desired false alarm probability. We prove that the detection probability of cooperative sensing can be maximized by optimally selecting the number of users with high INR and SNR. 
The remainder of this paper is organized as follows. Section II describes the system model in consideration. Section III describes the proposed cooperative sensing scheme in the presence of imperfect reporting channel and INR. Section IV verifies the performance of the proposed scheme by computer simulation. Finally, conclusions are given in Section V.

\section{SYSTEM MODELING}

\subsection{Single User Channel Sensing}

We consider a cognitive radio system where $K$ active users share a wideband channel comprising $M$ non-overlapped subchannels. Let $h_{k, m}$ be the channel between the primary user and secondary user $k$ at subchannel $m$. Then, user $k$ can detect the presence of the primary signal on subchannel $m$ by means of a simple the hypothesis test represented as

$$
\begin{aligned}
& \mathcal{H}_{0}: r_{k, m}=w, \\
& \mathcal{H}_{1}: r_{k, m}=h_{k, m} s_{m}+w,
\end{aligned}
$$

where $\mathcal{H}_{0}$ and $\mathcal{H}_{1}$ denote the hypothesis corresponding to the absence and the presence of the primary user, respectively, $r_{k, m}$ is the received signal of user $k$ through subchannel $m, s_{m}$ is the primary user's signal transmitted through subchannel $m$, and $w$ denotes zero mean Gaussian noise with variance $\sigma_{w}^{2}$.

We consider the use of an energy detector for the spectrum sensing. For subchannel $m$, the test statistic of user $k$ for the hypothesis test can be given by [4]

$$
Y_{k, m}=\sum_{l=0}^{L-1}\left|r_{k, m}(l)\right|^{2},
$$

where $L$ denotes the number of samples. Then, user $k$ detects the existence of the primary user's signal on subchannel $m$ by

$$
\varphi_{k, m}(n)= \begin{cases}0, & Y_{k, m}<\lambda, \\ 1, & Y_{k, m} \geq \lambda,\end{cases}
$$

where $\lambda$ is a threshold value to be determined. Letting $\eta_{k, m}$ be the INR of user $k$ at subchannel $m$, it can be shown that the test statistic $Y_{k, m}$ is asymptotically normally distributed with mean [6]

$$
E\left[Y_{k, m}\right]= \begin{cases}L \sigma_{w}^{2}, & \mathcal{H}_{0} \\ \left(L+\eta_{k, m}\right) \sigma_{w}^{2}, & \mathcal{H}_{1}\end{cases}
$$

and variance

$$
\operatorname{Var}\left[Y_{k, m}\right]= \begin{cases}2 L \sigma_{w}^{2}, & \mathcal{H}_{0} \\ 2\left(L+2 \eta_{k, m}\right) \sigma_{w}^{2}, & \mathcal{H}_{1} .\end{cases}
$$

Thus, user $k$ detects the existence of the primary user's signal on subchannel $m$ with false alarm and detection probability given by, respectively, [6]

$$
\begin{gathered}
P_{f}^{k, m}=\operatorname{Pr}\left(Y_{k, m}>\lambda \mid \mathcal{H}_{0}\right)=Q\left(\frac{\lambda-L \sigma_{w}^{2}}{\sqrt{2 L} \sigma_{w}^{2}}\right) \\
P_{d}^{k, m}=\operatorname{Pr}\left(Y_{k, m}>\lambda \mid \mathcal{H}_{1}\right)=Q\left(\frac{\lambda-L \sigma_{w}^{2}\left(1+\eta_{k, m}\right)}{\sigma_{w}^{2} \sqrt{2 L\left(1+2 \eta_{k, m}\right)}}\right),
\end{gathered}
$$

where $Q(\cdot)$ is the complementary cumulative distribution function of a zero mean Gaussian random variable with unit variance.

\subsection{Cooperative Channel Sensing}

For cooperative sensing, the BS receives single-bit decision $\left\{\varphi_{k, m}\right\}$ of all users. Let $\tilde{\varphi}_{k, m}$ be the received value of a single-bit decision $\varphi_{k, m}$ from user $k$. Assuming that $C(\leq K)$ users are in cooperation, the existence of the primary user's signal on subchannel $m$ can be detected by

$$
\begin{aligned}
& \mathcal{H}_{0}: \sum_{k=0}^{C-1} \tilde{\varphi}_{k, m}<N \\
& \mathcal{H}_{1}: \sum_{k=0}^{C-1} \tilde{\varphi}_{k, m} \geq N .
\end{aligned}
$$

When the OR rule (i.e., $N=1$ ) is applied, the corresponding false alarm and detection probability at subchannel $m$ are respectively represented as [6]

$$
\begin{aligned}
& P_{f}^{m}(C)=1-\prod_{k=1}^{C}\left(1-P_{f}^{k, m}\right) \\
& P_{d}^{m}(C)=1-\prod_{k=1}^{C}\left(1-P_{d}^{k, m}\right) .
\end{aligned}
$$

\section{Proposed Cooperative Spectrum Sensing}

In cognitive radio systems, high detection probability may yield low interference to the primary user and low false alarm probability may improve the spectrum efficiency. Since these two probabilities are conflicting to each other, it may be desirable to employ a sensing scheme that yields high detection probability while satisfying a desired false alarm probability. We consider the design of a detection scheme that maximizes the detection probability with a constraint on the false alarm probability as

$$
\max _{C} P_{d}^{m}(C) \text { subject to } P_{f}^{m}(C) \leq \alpha .
$$

Since (11) is a function of $C$, we find the optimum number of users that maximizes the detection probability for a given false alarm probability. To this end, we analyze the detection probability according to the number of cooperating users with and without the presence of channel errors for the reporting.

\subsection{Optimization without Channel Error}

Let $\bar{P}_{f}^{m}\left(=\alpha^{\prime} \leq \alpha\right)$ be the desired false alarm probability at subchannel $m$. Assuming that all the decisions are reported to the BS without channel error (i.e., $\tilde{\varphi}_{k, m}=\varphi_{k, m}$ ), it can be shown 
from (9) that the required false alarm probability of user $k$ is given by

$$
\bar{P}_{f}^{k, m}=1-\sqrt[C]{1-\alpha^{\prime}}
$$

It can be shown from (6) that the threshold for the corresponding false alarm probability is determined by

$$
\lambda=Q^{-1}\left(1-\sqrt[C]{1-\alpha^{\prime}}\right) \sigma_{w}^{2} \sqrt{2 L}+\sigma_{w}^{2} L
$$

Thus, the detection probability of user $k$ at subchannel $m$ is given by

$$
P_{d}^{k, m}=Q\left(\frac{1}{\sqrt{1+2 \eta_{k, m}}}\left(Q^{-1}\left(1-\sqrt[C]{1-\alpha^{\prime}}\right)-\sqrt{\frac{L}{2}} \eta_{k, m}\right)\right) .
$$

It can be seen that the larger $\eta_{k, m}$, the larger $P_{d}^{k, m}$. Thus, it is desirable for the maximization of the detection probability to select users with high INR for the cooperation. Without loss of generality, assuming that $\eta_{1, m}>\eta_{2, m}>\ldots>\eta_{K, m}$, the detection probability at subchannel $m$ can be represented as

$$
P_{d}^{m}(C)=1-\prod_{k=1}^{C}\left(1-Q\left(\frac{1}{\sqrt{1+2 \eta_{k, m}}}\left(\begin{array}{l}
Q^{-1}\left(1-\sqrt[C]{1-\alpha^{\prime}}\right) \\
-\sqrt{\frac{L}{2}} \eta_{k, m}
\end{array}\right)\right)\right)
$$

As the number $C$ increases, $P_{d}^{k(\leq C-1), m}$ decreases due to the term $Q^{-1}\left(1-\sqrt[C]{1-\alpha^{\prime}}\right)$ in (14), where $k \leq C-1$. This implies the increase of threshold $\lambda$ since $\bar{P}_{f}^{k, m}$ decreases as $C$ increases. This implies that the cooperation of all $K$ users for the spectrum sensing does not necessarily achieve the optimum performance. The optimum number $\hat{C}$ for the cooperation can be determined by (15) for a given $\eta_{k, m}$.

\subsection{Optimization with Channel Errors}

In practice, some decision results can be reported to the BS with errors (i.e., $\tilde{\varphi}_{k, m} \neq \varphi_{k, m}$ for some $k$ ). Assuming that the channel of user $k$ transmits data with a bit error rate (BER) of $P_{e}^{k}$, the detection and false alarm probability at subchannel $m$ can be represented as, respectively,

$$
\begin{aligned}
& P_{d}^{m}(C)=1-\prod_{k=1}^{C}\left[\left(1-P_{d}^{k, m}\right)\left(1-P_{e}^{k}\right)+P_{d}^{k, m} P_{e}^{k}\right] \\
& P_{f}^{m}(C)=1-\prod_{k=1}^{C}\left[\left(1-P_{f}^{k, m}\right)\left(1-P_{e}^{k}\right)+P_{f}^{k, m} P_{e}^{k}\right] .
\end{aligned}
$$

Assume that each user reports its decision results to the BS through a subchannel in the best condition to minimize the reporting error. Letting $\hat{m}_{k}$ be the index of the subchannel of user $k$ in the best condition, i.e.,

$$
\hat{m}_{k}=\arg \max _{m}\left\{\gamma_{k, m}\right\},
$$

the probability density function of $\gamma_{k, \hat{m}_{k}}$ can be represented as

$$
f(x)=\frac{M}{\bar{\gamma}_{k}} e^{-\frac{x}{\bar{\gamma}_{k}}}\left(1-e^{-\frac{x}{\bar{\gamma}_{k}}}\right)^{M-1}
$$

where $\gamma_{k, m}$ denotes the SNR of user $k$ at subchannel $m$ and $\bar{\gamma}_{k}$ is the average SNR of user $k$, given by $\bar{\gamma}_{k}=\sum_{m=1}^{M} \gamma_{k, m}$.

When the decision result is reported by means of BPSK signaling, it can be shown that the BER for a given $\gamma_{k, \hat{m}_{k}}$ is given by

$$
P_{e \gamma_{k, \hat{m}_{k}}^{k}}^{k}=Q\left(\sqrt{2 \gamma_{k, \hat{m}_{k}}}\right)
$$

Then, the average BER in Rayleigh fading channel is

$$
\begin{aligned}
P_{e}^{k} & =\int_{0}^{\infty} P_{e \mid \gamma_{k, \hat{m}_{k}}^{k}}^{k} f\left(\gamma_{k, \hat{m}_{k}}\right) d \gamma_{k, \hat{m}_{k}} \\
& =\sum_{m=0}^{M-1}\left(\begin{array}{c}
M-1 \\
m
\end{array}\right)(-1)^{M-m-1} \frac{M}{2(M-m)}\left(1-\sqrt{\frac{\bar{\gamma}_{k}}{M-m+\bar{\gamma}_{k}}}\right) .
\end{aligned}
$$

Taking account of the channel error, the required false alarm probability of user $k$ is given by

$$
\bar{P}_{f}^{k, m}=\frac{P_{e}^{k}-1+\sqrt[C]{1-\alpha^{\prime}}}{2 P_{e}^{k}-1}
$$

and the corresponding threshold value is

$$
\lambda_{k}=Q^{-1}\left(\frac{P_{e}^{k}-1+\sqrt[C]{1-\alpha^{\prime}}}{2 P_{e}^{k}-1}\right) \sigma_{w}^{2} \sqrt{2 L}+\sigma_{w}^{2} L
$$

Unlike in the case of no reporting error, the threshold for the decision depends on $P_{e}^{k}$ of each user. It can be shown from (7) that the detection probability of user $k$ is given by

$$
P_{d}^{k, m}=Q\left(\frac{1}{\sqrt{1+2 \eta_{k, m}}}\left(Q^{-1}\left(\frac{P_{e}^{k}-1+\sqrt[C]{1-\alpha^{\prime}}}{2 P_{e}^{k}-1}\right)-\sqrt{\frac{L}{2}} \eta_{k, m}\right)\right)
$$

Notice that as the number $C$ increases, $P_{d}^{k(\leq C-1), m}$ decreases due to the term $Q^{-1}\left(\left(P_{e}^{k}-1+\sqrt[C]{1-\alpha^{\prime}}\right) /\left(2 P_{e}^{k}-1\right)\right)$. This implies that $1 \leq \hat{C} \leq K$. 
TABLE I

SIMULATION PARAMETERS

\begin{tabular}{cc}
\hline \hline PARAMETERS & Values \\
\hline Noise level & $25 \mathrm{dBm}$ \\
The number of subchannels $(M)$ & $-114 \mathrm{dBm}$ \\
Bandwidth of subchannels & 40 \\
Path loss model & $1 \mathrm{MHz}$ \\
Shadowing model & COST 231 - Hata Suburban [8] \\
\hline \hline
\end{tabular}

If the detection probability of all users is the same (i.e., INR and SNR of all users are the same), the optimum number $\hat{C}$ is simply equal to $K / 2$ [7]. If not, it is not easy to determine $\hat{C}$ in a simple function of $K$ due to the involvement of Q-function [7]. We determine the optimum number $\hat{C}$ from (16) and (24) for given $\eta_{k, m}$ and $\bar{\gamma}_{k}$ by an empirical method as follows:

Step I: Initialize the maximum detection probability $\hat{P}_{d}^{m}$

$$
\hat{P}_{d}^{m}=P_{d}^{m}(1)=P_{d}^{1, m}+P_{e}^{1}-2 P_{d}^{1, m} P_{e}^{1} \text { and } C=2 .
$$

Step II: Initialize a set $\Phi_{C}$ of $C$ users selected for the cooperation

$$
\Phi_{C}=\varnothing, \Theta_{1}=\{1,2, \ldots, K\} \text { and } i=1 .
$$

Step III: Select a user with the smallest $\left(1-P_{d}^{k, m}\right)\left(1-P_{e}^{k}\right)$ $+P_{d}^{k, m} P_{e}^{k}$ and then update $\Phi_{C}$ as

$$
\begin{aligned}
& \text { for } \pi_{i}=\arg \min _{k \in \Theta_{i}}\left(1-P_{d}^{k, m}\right)\left(1-P_{e}^{k}\right)+P_{d}^{k, m} P_{e}^{k}, \\
& \Phi_{C} \leftarrow \Phi_{C} \cup \pi_{i} \text { and } \Theta_{i+1}=\Theta_{i}-\pi_{i} .
\end{aligned}
$$

Step IV: If $\left|\Phi_{C}\right|<C$, then $i \leftarrow i+1$ and go to step II. Else calculate the $P_{d}^{m}(C)$ as

$$
P_{d}^{m}(C)=1-\prod_{k=1, k \in \Phi_{C}}^{K}\left(1-P_{d}^{k, m}\right)\left(1-P_{e}^{k}\right)+P_{d}^{k, m} P_{e}^{k}
$$

Step V: If $P_{d}^{m}(C)>\hat{P}_{d}^{m}$, then $\hat{P}_{d}^{m}=P_{d}^{m}(C), C \leftarrow C+1$ and go to step II. Else stop.

The maximum detection probability at subchannel $m$ for a given $\Phi_{\hat{C}}$ can be represented as

$$
\hat{P}_{d}^{m}=1-\prod_{k=1}^{\hat{C}}\left(1-Q\left(\frac{1}{\sqrt{1+2 \eta_{k, m}}}\left(\begin{array}{l}
Q^{-1}\left(1-\sqrt[\hat{C}]{1-\alpha^{\prime}}\right) \\
-\sqrt{\frac{L}{2}} \eta_{k, m}
\end{array}\right)\right)\right)
$$

for $k \in \Phi_{\hat{C}}$.
In summary, the overall procedure can be described as follows:

1. Each user reports the INR and SNR to the BS.

2. The BS determines a set of optimum users by (24).

3. Selected users make their decision by (3) with a threshold determined by (23) and report their decision to the BS.

4. Finally, the BS makes the final decision on subchannel $m$ by (8).

\section{Performance Evaluation}

We verify the performance of the proposed scheme by computer simulation. We assume that a macro-cell base station is the primary station, a femtocell BS is the secondary station and femtocell mobile stations are the secondary users. We also assume that users are uniformly distributed within the coverage $40 \mathrm{~m}$ of the secondary BS and the secondary BS is $800 \mathrm{~m}$ away from the primary user, unless explicitly stated otherwise. To verify the validation of the proposed scheme, we compare the performance of the proposed scheme with that of all-user cooperation scheme. The performance of a single user sensing scheme is also considered for reference. The desired false alarm probability is set to 0.01 for the simulation. To satisfy the constraint for the false alarm probability, we assume that all the schemes determine the decision threshold by (23).

Fig. 1 depicts the detection probability of the proposed scheme according to the user SNR when $K=50$, and $M=20,30$ and 40. We assume that the SNR of each user is the same to verify the effect of the reporting error. It can be seen that as the user SNR increases, the optimum detection probability $\hat{P}_{d}^{m}$ increases. This is mainly due to the fact that the larger the SNR, the less the reporting error. When the number $M$ of subchannels is 40 , it can be seen that the best detection performance is achieved. This is due to the fact that as the number of subchannels increases, the frequency diversity effect makes the reporting error decrease. It can also be seen that $\hat{P}_{d}^{m}$ is limited to 0.93 even though the SNR is sufficiently high. This is due to the fact that the reporting errors do not occur for all $M$ when the SNR is higher than $5 \mathrm{~dB}$ and the number $K$ of users for the cooperation is set to 50 .

Fig. 2 depicts the detection probability of the proposed scheme according to the number $C$ of cooperating users when $K=30,50$ and 100 . It can be seen that the detection probability increases rapidly to a peak point and then decreases gradually as $C$ increases. This verifies that the cooperation among all users is not optimum. It can also be seen that as the number of users in the network increases, the optimum detection probability $\hat{P}_{d}^{m}$ increases. This is mainly due to the fact that the number of users with high $\eta_{k, m}$ and $\bar{\gamma}_{k}$ increases as $K$ increases. 


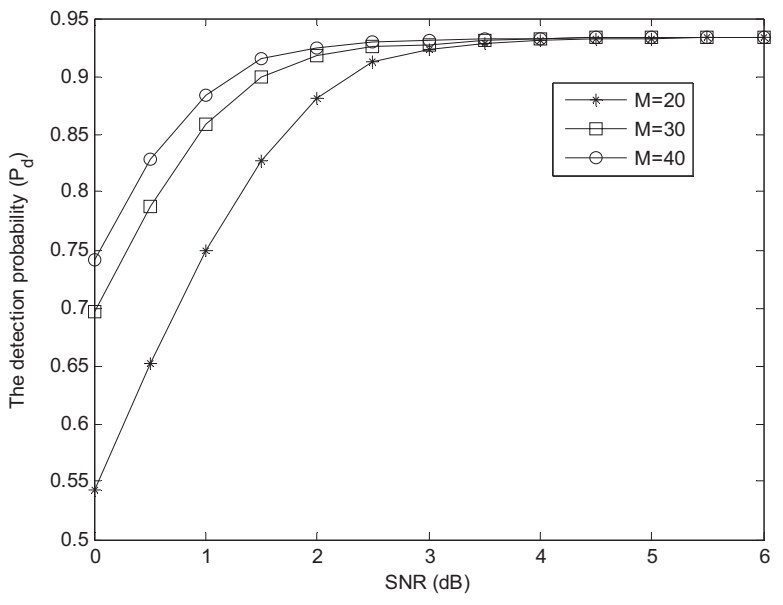

Figure 1 . The detection probability according to $\gamma_{k, m}$ when $K=50$, $M=20,30$ and 40

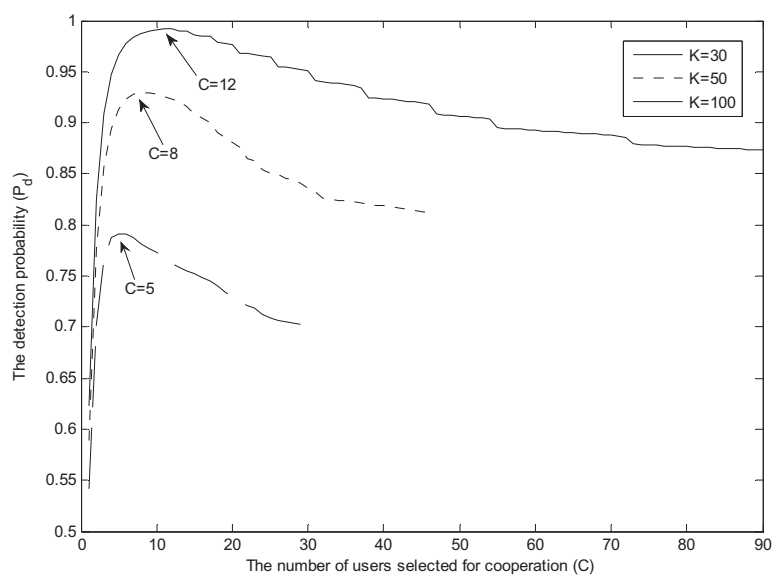

Figure 2. The detection probability according to $C$ when $K=30,50$ and 100 .

Fig. 3 depicts the detection probability of the proposed scheme in terms of the number of users in the network. It can be seen that the proposed scheme significantly outperforms the other schemes. It can also be seen that the single user sensing scheme slightly outperforms the all-user cooperation scheme when $K<14$. This is because the optimum number $\hat{C}$ for the cooperation is 1 or 2 when $K<14$.

\section{Conclusions}

We have considered cooperative spectrum sensing in cognitive radio systems by linearly combining hard-decision of each user. The proposed scheme maximizes the detection probability by optimally finding cooperating users for a given false alarm probability. The analytic and simulation results show that cooperative sensing among users with high INR and SNR can provide higher detection probability than that among all users in the network, and that single user sensing may outperform cooperative sensing when the number of users in the network is small.

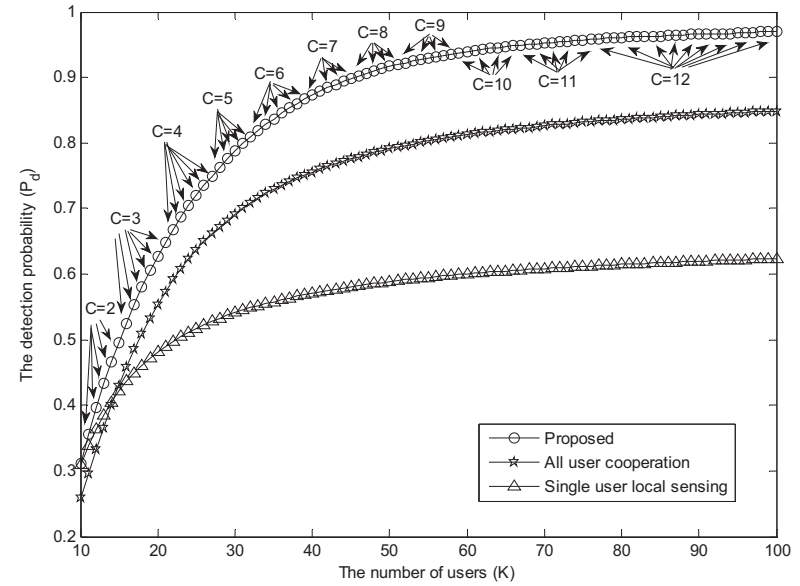

Figure 3 . The detection probability according to $K$.

\section{ACKNOWLEDGMENTS}

This work was supported by the Seoul R\&BS Program (10544).

\section{REFERENCES}

[1] A. Ghasemi and E.S. Sousa, "Opportunistic spectrum access in fading channels through collaborative sensing," IEEE Journal of Commun., vol. 2, no. 2, pp. 1932-1940, Mar. 2007.

[2] G. Ganesan and Y. G. Li, "Cooperative spectrum sensing in cognitive radio, part 1: two user networks," IEEE Trans. Wireless Commun., vol. 6, no. 6, pp. 1536-1544, June 2007.

[3] Y. Yu, H. Murata and S. Yoshida, "Interference information based power control for cognitive radio with multi-hop cooperative sensing," IEICE Trans. Commun., vol. 91, no. 1, pp. 516-524, June 2008.

[4] D. Cabric, S. M. Mishra, and R. W. Brodersen, "Implementation issues in spectrum sensing for cognitive radios," in Proc. Asilomar Conf. Signals, Systems and Computers, pp. 7-10, Nov. 2004.

[5] V. I. Kostylev, "Energy detection of a signal with random amplitude," in Proc. IEEE Int. Conf. Commun. pp. 16061610, May 2002.

[6] A. Ghasemi and E. S. Sousa, "Spectrum sensing in cognitive radio networks: the cooperation-processing tradeoff," Wireless Commun. and Mobile Comput., vol. 7, no. 1, pp. 1049-1060, Sept. 2007.

[7] C. Sun, W. Zhang, and K. B. Letaief, "Cooperative spectrum sensing for cognitive radios under bandwidth constraints," in Proc. IEEE Wireless Commun. and Netw. Conf., pp. 25-30, Feb. 2007.

[8] W. Zhang and K. Letaief, "Cooperative spectrum sensing optimization in cognitive radio networks," in Proc. IEEE Int. Conf. Commun. pp. 3411-3415, May 2008.

[9] V. S. Abhayawardhana and I. J. Wassell, "Comparison of empirical propagation path loss models for fixed wireless access systems," in Proc. IEEE Veh. Technol. Conf., pp. 7377, June 2005. 